\title{
Rapid assessment of malaria risk using entomological techniques: taking an epidemiological snapshot
}

\author{
P.F. Billingsley ${ }^{1}$, J.D. Charlwood ${ }^{2}$ and B.G.J. Knols ${ }^{3,4}$
}

\begin{abstract}
The pivotal role of mosquitoes in malaria epidemiology means that in practice, control efforts against the disease must include sustainable control of the vectors. This has fostered many careful and complex longitudinal studies designed to estimate the entomological inoculation rate (EIR) as a measure of risk of exposure to infection. Alternatives to this estimate - anti-circumsporozoite protein antibodies, conversion rates in non-immunes or re-infection rates - require sampling of blood from patients and have associated compliance and interpretation issues. Here, we describe how entomological techniques, which provide information on mosquito population densities, age structures and infection characteristics, allied with mapping and modelling approaches can provide a relative estimate of EIR within a few days of fieldwork. The information could be used to decide upon and track intervention measures, as well as to monitor disease outbreaks. Standardizing the techniques used for rapid assessment could enable comparison of malaria risk in different ecological and epidemiological settings.
\end{abstract}

Keywords: malaria; mosquito; epidemiology; entomological infection rate, rapid assessment, transmission

\section{Introduction}

Time spent in reconnaissance is never wasted - this is as true for a great general such as Napoleon as for those involved in infectious disease control, and so the gathering of reliable information on exposure risk is a central facet of malaria control programmes. Reduction of exposure to the vectors, either by controlling mosquitoes or avoiding their bites, is still a major element in malaria control policies. However, local resources are usually severely restricted and administrators often have to make decisions based on limited information. Not only administrators but communities, families and individuals affected by the disease all need adequate information to decide where, when and what they should do to reduce the risk of infection.

In many ways malaria transmission patterns are like the fractals seen in chaos theory. The so-called ' $80 / 20$ rule' encapsulates the idea that most malaria

\footnotetext{
${ }^{1}$ School of Biological Sciences, University of Aberdeen, Tillydrone Avenue, Aberdeen AB24 2TZ, UK. E-mail: p.billingsley@abdn.ac.uk

${ }^{2}$ DBL - Institute for Health, Research and Development, DK 2920 Charlottenlund, Denmark

${ }^{3}$ Entomology Unit, Agency's Laboratories Seibersdorf, International Atomic Energy Agency, A-1400

Vienna, Austria

${ }^{4}$ Laboratory of Entomology, Wageningen University, PO Box 8031, 6700 EH Wageningen, The Netherlands
} 
transmission is concentrated in a small fraction of the population (Woolhouse et al. 1997). Thus, heterogeneous patterns of transmission rates are reproduced at differing levels of scale. For example, even within a high-transmission district some villages will have a greater amount of transmission than others. Within those villages most of the transmission will be restricted to a small number of hamlets, within hamlets to some houses and even within houses to some individuals (Smith et al. 1995).

Rapid assessment (RA) techniques aim to provide information at any or all of these different scales to identify areas where transmission is concentrated, enabling the optimal use of limited resources for control. Although RA cannot provide the same depth and breadth of data as longitudinal studies, the scope and reliability of the data it provides will increase with use and so its utility to health-planning operations should also grow. Before this happens, however, RA must become accepted as a viable addition, rather than alternative, to the classic longitudinal survey. In malaria epidemiology, longitudinal studies are commonly regarded as the essential prerequisite to planned intervention trials. Short-term studies are often decried because they may represent only a single transmission cycle, a single season or a single year, or have no local comparative data. Longitudinal studies can sometimes ignore the spatial variation of transmission within a study area (Thompson et al. 1997; Smith et al. 1995) and take a long time to provide answers. Thus, they become cumbersome and inappropriate when and where responsive control is needed in the face of changing malaria patterns or epidemic malaria emergence. For example, in refugee situations sometimes large numbers of people, from different epidemiological (immunological) backgrounds, set up temporary homes in areas where there may be a significant risk of transmission. In such a scenario, a protracted assessment would be unethical, but implementing a control programme without any assessment would have potential for not solving the problem.

Models for malaria transmission place great emphasis on the mosquito (Killeen et al. 2000b; Koella 1991), so information gathered about the vector is crucial. Gathering vector data is also less dependent upon human compliance. In this article we will demonstrate how information from mosquito collections and dissections might be considered sufficient to estimate relative exposure to malaria and hence provide estimates of malaria risk in different malaria epidemiological settings.

\section{Measuring risk}

There are a number of ways of estimating the risk to a non-immune or semiimmune individual of acquiring a malaria infection. The best indicator, and the only one suitable for use in high transmission areas, is the entomological inoculation rate (EIR), which is classically calculated from the number of mosquitoes biting an 'average' human host and the proportion of these that contain (infective) sporozoites (Garrett-Jones 1964). At low transmission rates, the measurement of anti-CSP antibodies in human hosts (Wijesundera et al. 1990) or of conversion rates (Gupta and Snow 1996) provides two alternatives to EIR estimations. However, when transmission rates increase above a threshold, correlations between these three parameters can be lost (Kilombero Malaria Project 1992). Where existing parasites have been cleared, the conversion rates have been considered an indicator of malaria risk (Trigg et al. 1997), but without the application of PCR-based techniques it is impossible to differentiate between re-infections and recrudescence of parasites suppressed by drugs or the immune system. It is usually argued that the best way of obtaining a picture of the epidemiology of malaria in a new setting is to conduct a 
cross-sectional parasite prevalence survey and for such surveys, dipstick assays can provide immediate results. While entomological and parasitological surveys can be performed simultaneously, in areas of high transmission it is likely that parasitaemias will be saturated and information on spatial distribution of risk of infection rather than parasites will not be obtainable from parasitological data alone.

There is a need to identify relative as well as absolute risk of infection. Using RA to decide upon suitable interventions may seem questionable since control efforts are not likely to be directed solely at the few high-density houses in a study site. We would argue however that, whilst not ignoring the rest of the area, interventions should initially be directed at or include such areas, be they houses in a village or villages in a district. Often the most exposed households are those in the more remote areas. Given the 80/20 rule, if these households are ignored or not identified prior to implementation, control will be incomplete and ineffective despite what might otherwise seem to be a good coverage. Thus, one purpose of the RA is to identify transmission 'hot spots' that should be prioritized for interventions. For example, in the Ifakara region of Tanzania, although the 'average' villagers may receive a mean of 300 infective bites per year, some villagers receive 10 times less than that and some, who live in the more remote areas, 10 times more, this heterogeneity being a direct consequence of variation in mosquito biting-rates (Figure 1) (Charlwood et al. 1995a; 1995b).

\section{Rapid assessment: snapshot epidemiology?}

\section{The pros and cons of rapid assessment}

A good RA will provide information about relative and perhaps absolute risk in a short time. It is likely to be most effective when deployed over a wide area, giving a series of 'snapshots' or stills from what can be considered a longitudinal 'film'. Increases in absolute (and sometimes relative) oocyst and sporozoite infections are the inevitable presages of malaria outbreaks. Where these can be identified prior to epidemics of human infection, and the risk of infection determined with some degree of accuracy, then pre-epidemic intervention is a conceivable outcome of RA. This holds true for a range of settings including epidemics in migrant or refugee populations, seasonal malaria transmission, where land use is changing, and where drug or insecticide resistance is developing. The general approach outlined below and in Figure 2 is applicable to any vector-borne disease where trapping, collecting and analysis of vectors are suitably advanced.

There are critical requirements for RA protocols that underpin any adaptations to the local situation. RA should be capable of collecting, processing and analysing the bulk of the necessary samples and data in the field, and thereby provide an acceptable and accurate estimate of the malaria risk. Like any assay, entomology-led RA needs to be sensitive, since assessments might be undertaken at low mosquito population densities. Nevertheless, there will be some sites and times of year when vector populations are so small that RA of mosquito populations, particularly adults, is just not feasible. While accepting these caveats' broad applicability, flexibility and ease of use will be important in the acceptance of RA. With field data collected and analysed 


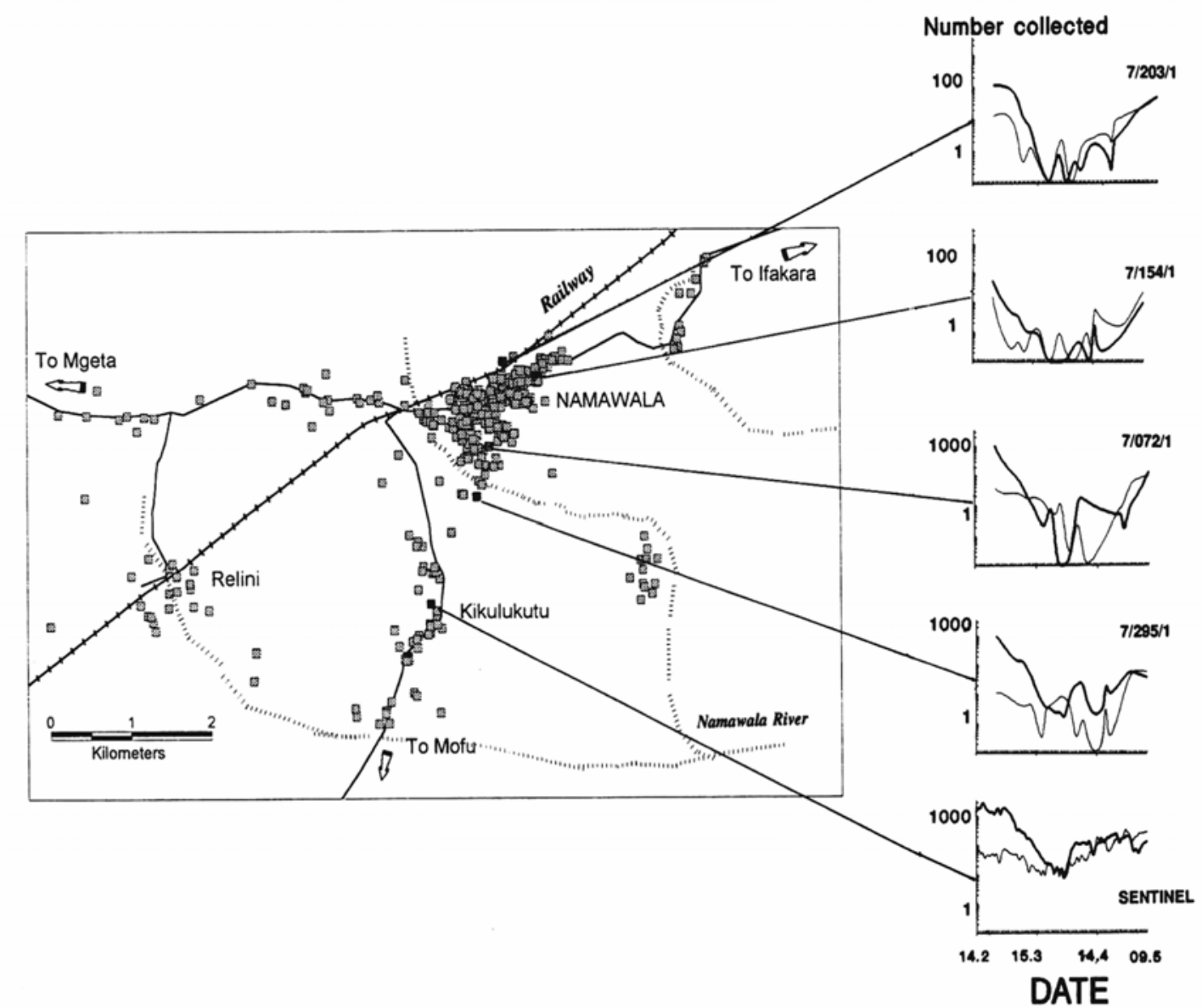

Figure 1. Spatial heterogeneity of mosquito numbers in an African village. CDC light traps were used to collect Anopheles gambiae s.l. (heavy lines) and An. funestus (thin lines) in houses in and around the village of Namawala, Tanzania in February to May 1991. The map shows the relative positions of the houses, and the graphs show that, although absolute numbers are different at each site, the ratio of each species between houses stays relatively constant. Using a sentinel light trap in an area of high mosquito density allows immediate mapping and comparison of spatially heterogeneous data. Reprinted from Charlwood et al. (1995a; 1995b) with permission from CABI publishing

in consistent and comparable ways, there is also the exciting possibility that information can be collated from diverse ecological and epidemiological settings, contributing the much-needed fine-scale resolution for national-, regional- and continental-scale estimates of malaria risk (such as Mapping Malaria Risk in Africa) (Le Sueur et al. 1997).

\section{Background information}

Although there are few malaria-endemic regions where mosquito species and populations have not been described, priming on local species complexes, transmission rates, exo- or endophilic mosquito behaviour, and presence of each Plasmodium species will be essential. On-line databases can provide time-saving and 




Figure 2. Proposed outline for rapid assessment of malaria. The process for the RA from inception to completion is shown in the shaded boxes (centre column). The process requires input of information and its modification to suit the local situation at all stages of the RA (left shaded area). Similarly, there is a series of rational decisions to be made that will influence the scale, start and end of the RA (right shaded column). The completed RA report will vary to suit the sponsors and the objectives they have set, but in all cases a clear weighting should be given to the completed RA

decision-enhancing information. Amongst these, the normalized vegetation index (NDVI) can allow the presence of members of a species complex to be predicted with some accuracy (Figure 3a) (Lindsay, Parson and Thomas 1998). This is clearly demonstrable for the Anopheles gambiae s.s. and An. arabiensis species where, if NDVI predicts high proportion of either species, the accuracy of that prediction is very high (Figure 3b).

However, the local microepidemiology is likely to differ from regional descriptions, and the RA team should be prepared for such eventualities. Setting the RA into a seasonal context is important since rainfall or NDVI may also be good 

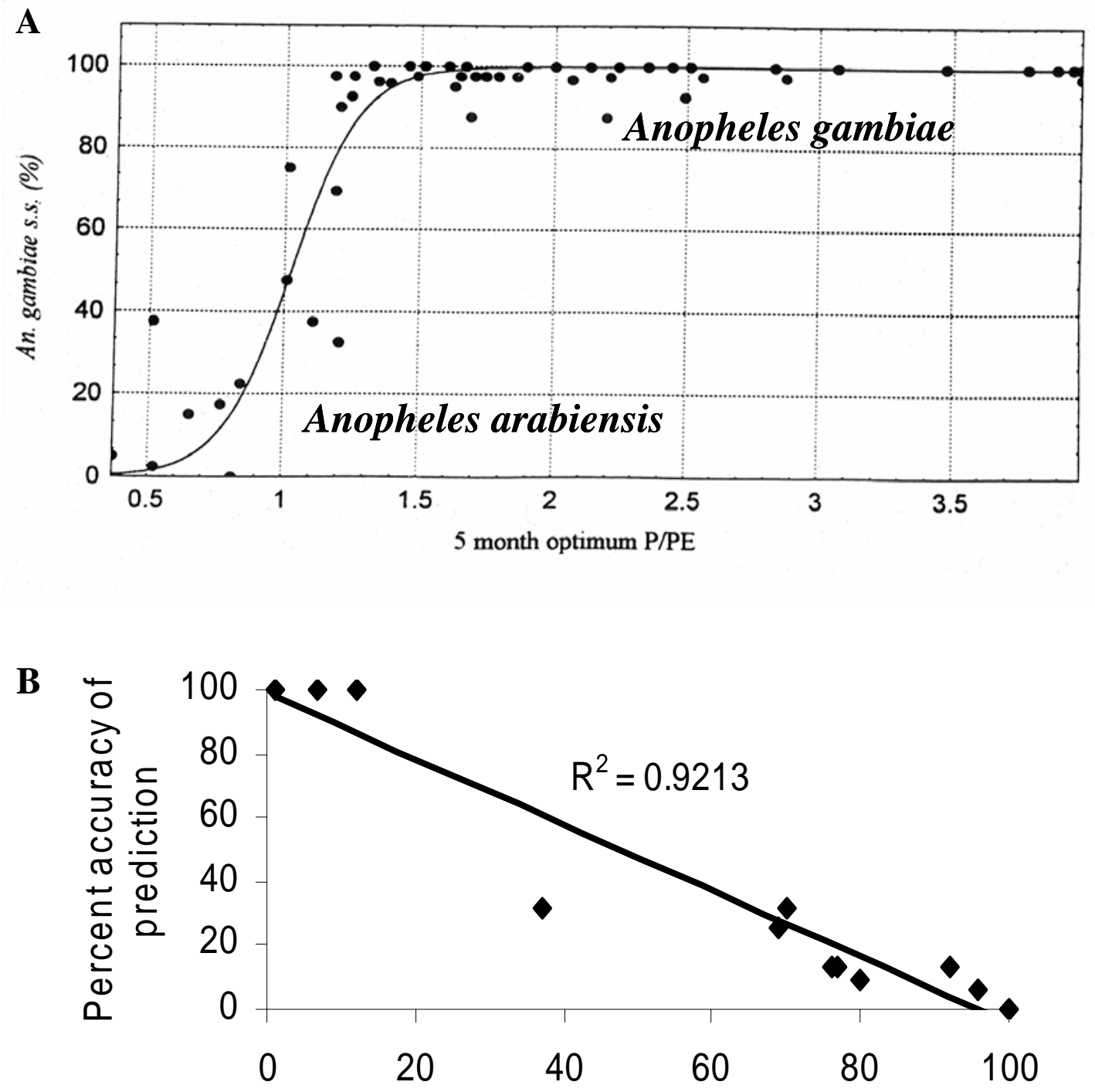

Percent A. gambiae expected

Figure 3. Prediction of relative Anopheles gambiae s.s. and Anopheles arabiensis based upon climate data. Measures of humidity (P/PE) were plotted against the proportion of each species at sites in Africa (A). An. gambiae s.s. and An. arabiensis separated based upon high $(\mathrm{P} / \mathrm{PE}>1.2)$ or low $(\mathrm{P} / \mathrm{PE}<0.8)$ humidity, respectively (courtesy of Prof. S. Lindsay, University of Durham, UK). The fitted line was used to predict the proportion of An. gambiae at other sites with good accuracy (B) especially at the extremes where populations of only one species would be expected (based on data in Lindsay, Parson and Thomas 1998)

predictors of mosquito numbers (Thomson et al. 1996; 1997) and population structures (Figure 4). At the start of the RA, non-entomological background data can be gained quickly. These can include information about human populations, living conditions and agricultural practices (most notably animal husbandry and those that favour vector breeding). Use of anti-mosquito measures such as bednets, mosquito coils, insecticides and repellents - all of which may be seasonally influenced (Charlwood et al. 1995b) - should be recorded. Simple questionnaires can also provide useful indicators on such things as anti-malarial drug usage, where people 
perceive mosquito populations to be high and of the presence of standing water (Teuscher 1992; Minja et al. 2001). In addition, recruitment of local expertise into the RA should excite local interest and provide opportunities for health education.

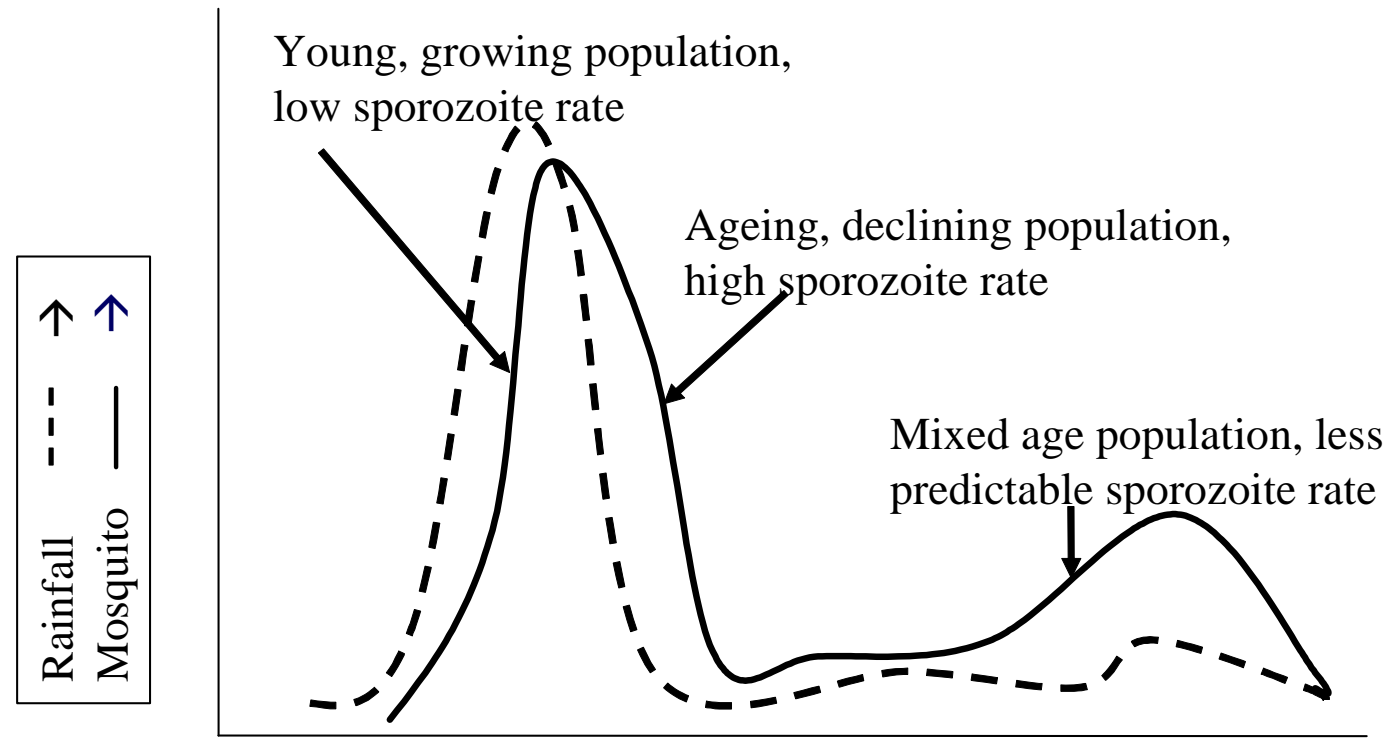

\section{Time of year $\rightarrow$}

Figure 4. Effects of season on mosquito populations and malaria transmission. Mosquito populations typically lag slightly behind rainfall, such that rainfall increases are often followed by a peak in mosquito numbers (see also Figure 1). Leading up to the peak, mosquito populations are undergoing huge recruitment, will be relatively young and therefore have low salivary-gland infection rates with Plasmodium sporozoites. As the population declines, the ageing mosquito population has few if any new recruits and will have an increasingly high sporozoite rate. During periods where the mosquito populations are more stable (short rains for example), the age of the mosquito population can be very mixed and the sporozoite infection rate hard to predict. RA undertaken at any of these times must account for the differences in mosquito population characteristics as well as densities that seasonality will induce

What type of data and how many?

The RA will have specific objectives to address that may be determined by outside agencies prior to fieldwork. Mosquitoes possibly represent the most captured insect group in the world, and many methods to catch them have been devised (Figure 5), from which several can be selected that best answer the specific questions. For example, if the aim is to determine spatial variations in the EIR, then adult mosquitoes from light traps, tent or bednet traps (Mathenge et al. 2002; 2004), or landing or resting catches will suffice. If parasitaemias or human to mosquito transmission are of interest then mosquitoes with blood meals need to be collected. Where mosquito feeding-strategies and population structure are of interest in relation to control measures such as bednet introduction, then live mosquitoes from diverse collections are most appropriate. If the purpose is to incriminate a vector, then the RA must be able to identify local mosquitoes and collect numbers sufficient for parasite identification possibly at low prevalences (Hii et al. 2000). Here, too, the seasonal timing of the RA will be important. Mosquito populations differ not only in density 


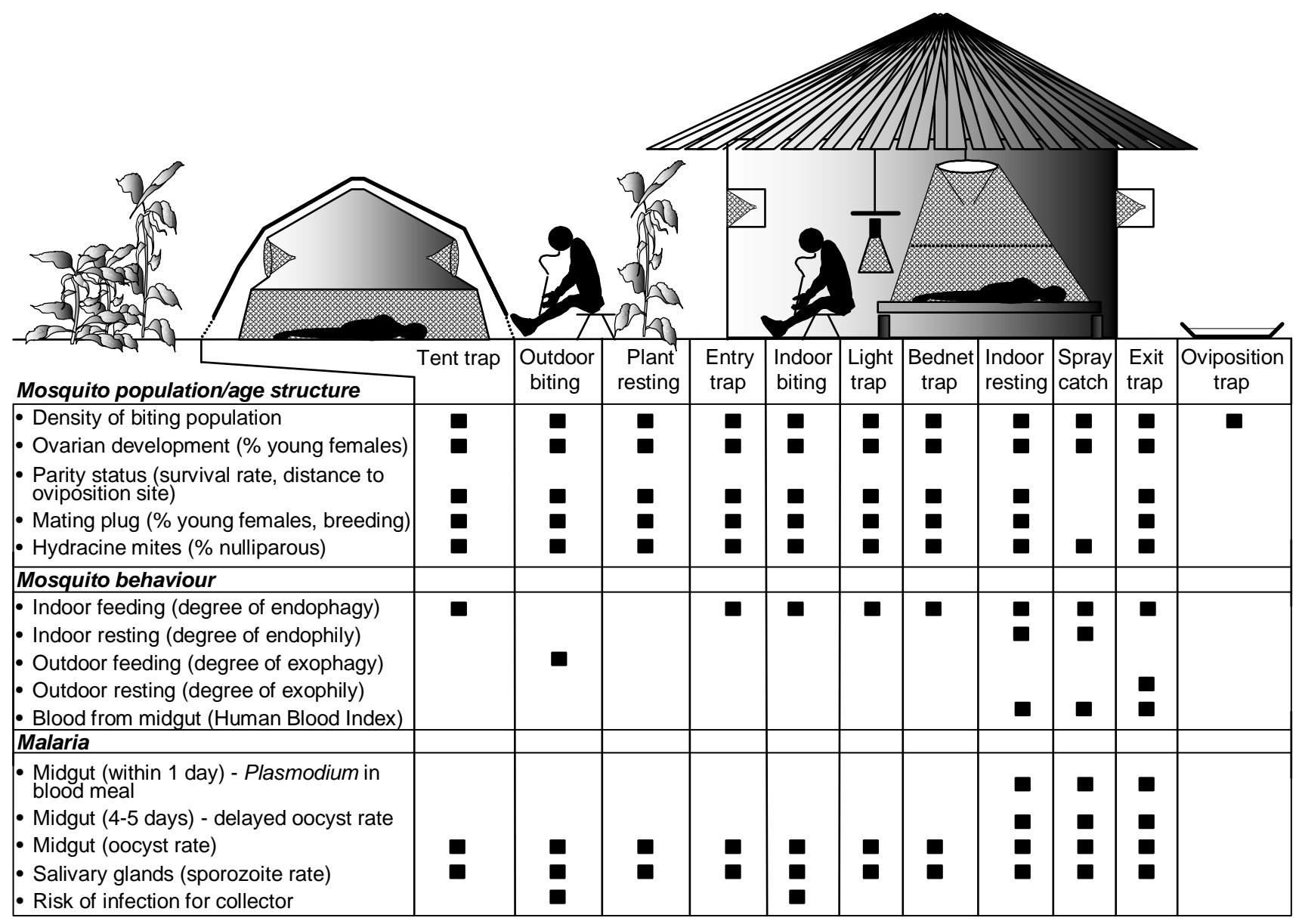

Figure 5. Mosquito-trapping methods and their application. Trapping techniques can gather information required for determining the EIR. Each trapping or collecting method and subsequent handling of the mosquitoes can be used to sample a definable subpopulation of the local mosquitoes (left column), and these are selected according the specific aims of the RA. For example, indoor resting catches will provide the full range of information necessary to assess mosquito population structure, association with human hosts, and essential malaria information, and up to six of these collections could be performed by a field worker per day 
but also in age structure, depending upon the season, and the RA should take into account the possibility that a population is stable, recruiting or in decline (Figure 1) as this greatly influences the sporozoite rate and EIR (Figure 4) (Charlwood et al. 1995a).

The more difficult decisions associated with RA are concerned with sampling effort - how many data are enough? When mosquito numbers are high, the job of the RA entomologist is much more straightforward. Mosquito densities will be collected and mapped quickly and with low effort, and estimating risk will be relatively routine for an experienced person. At low mosquito densities, decisions concerning the sampling effort will be crucial in determining the sensitivity of the RA. If several houses are sampled fruitlessly for mosquitoes, how is it possible to estimate risk? Lindblade, Walker and Wilson (2000) have addressed this question by comparing different sampling approaches and subsequent analyses to obtain estimates of risk of a malaria epidemic. If one uses a statistical approach driven by confidence intervals, then clearly the reliability of the data increases with sampling effort (20\% error in risk estimation for 102 houses sampled increasing to $50 \%$ error for 16 houses). A useful alternative is a sequential approach that essentially devolves the decision-making process about sampling effort to the entomologist in the field. The relationship between number of households sampled and number of mosquitoes found can be considered a measure of risk at low densities (Figure 6), and as sampling proceeds, the decision can be taken that sufficient households with mosquitoes have been identified (or not) to advise on control measures. Obviously it takes more sampling effort to determine no risk (50 households) than placing a site above a risk threshold (20 houses). Another measure that may prove useful is to estimate the probability of finding a mosquito within a certain sampling effort. For the low mosquito numbers sampled by Lindblade, Walker and Wilson (2000), there was a 95\% probability of finding a female An. gambiae in every seven houses sampled. However, the usefulness of these approaches remains largely untested, and the feasibility of their use in the field in control decision-making needs further consideration. Conversely, the over-dispersed pattern of oocyst infections in mosquitoes (Medley et al. 1993) has enabled the definition of a recognized minimum sampling effort (ca. 30 mosquitoes per sample) to estimate oocyst infection rates within predefined degrees of confidence (Billingsley et al. 1994).

An alternative and powerful approach is to exploit one or more of the many epidemiological models for malaria as an advisory tool for sampling strategies and effort. For example, the ratio of human to mosquito population densities, mosquito lifetime infectiousness and human infectious reservoir can be used in a relatively simple model for estimating EIR and the impacts of control measures upon it (Killeen et al. 2000b; 2000a). All of these parameters are measurable in the described RA approach. A still more powerful approach is the exploitation of less well-related datasets within a Bayesian statistical modelling approach (Smith et al. 1995). Bayesian modelling has been used to predict accurately $(86 \%)$ the number of villages in an Amazonian region at high risk of Onchocerca volvulus infections, and thereby offer advice on control efforts (Carabin et al. 2003). The flexibility of the Bayesian method and its ability to incorporate diverse data into a predictive, testable model make this a powerful approach that remains underused. We anticipate that it will become an integral part of RA. 


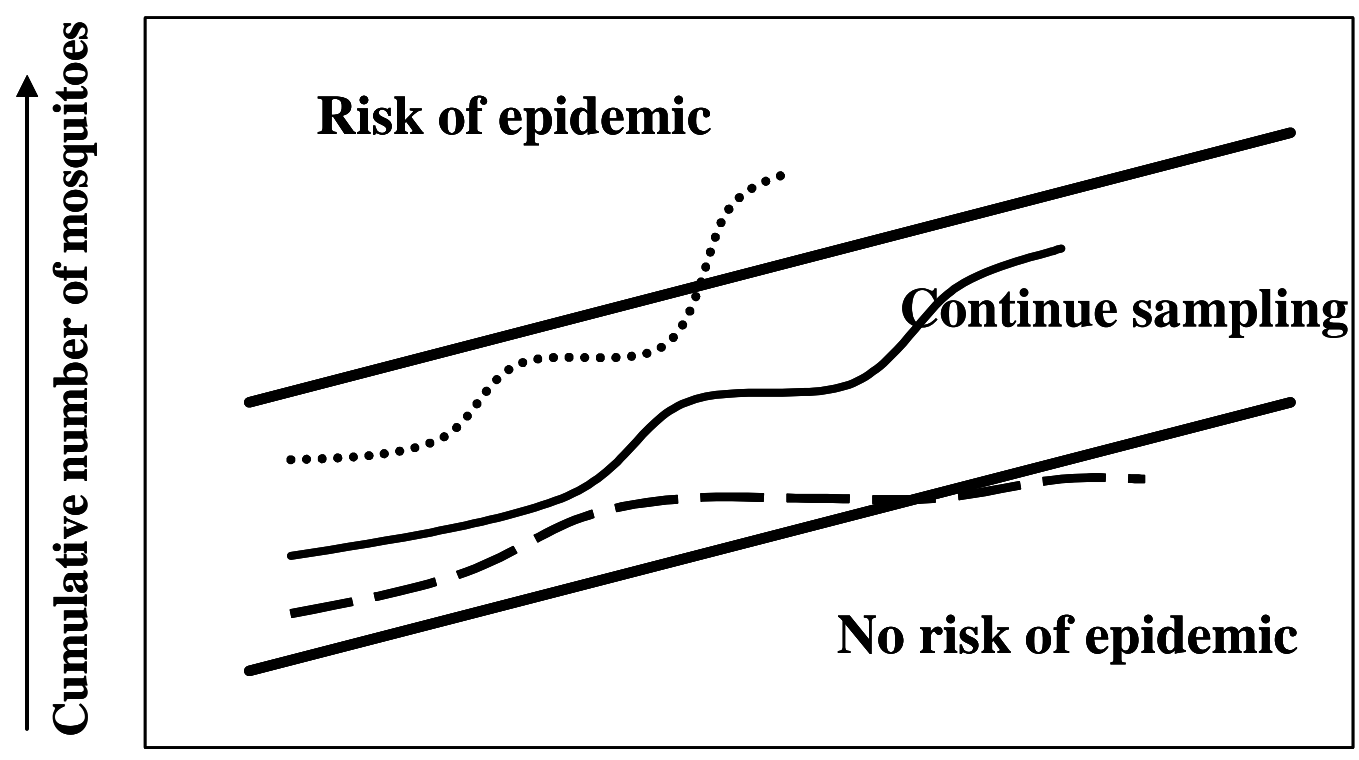

\section{Number of collections}

Figure 6. Trapping effort and outcome for malaria. The amount of effort that must be put into sampling has been modelled (Lindblade, Walker and Wilson 2000). The effort required is a combination of the required decision and the population densities of mosquitoes. As the number of samples accumulates, then the rate at which mosquitoes are found will help decide upon the risk to the community of a malaria epidemic. If sufficient mosquitoes are found with relatively low sampling effort ( ........... ), then epidemic risk can be considered high; where most samples do not contain mosquitoes ( - - - ) risk is low and sampling can stop. Otherwise, more sampling is required ( - ) in order to reach a decision about risk and therefore control. This open-ended approach is especially sensitive in areas where mosquito numbers are low and malaria is unstable

\section{Sample- and data-processing}

\section{Exploiting spatial information}

The spatial heterogeneity of mosquito populations can be used by RA to provide relative-risk maps of the sampled area (Ribeiro et al. 1996). The important first is the production of physical maps of the assessment area. Maps can be produced, and houses numbered, using a variety of techniques. Simple sketch maps can be used to localize each mosquito collection site and provide crude reference points. The integration of RA maps into a larger frame of reference requires at least two geopositions obtained either from 1:10,000 maps or from hand-held Global Positioning Satellite (GPS) devices. Well-produced maps are useful investments for future reference and therefore must incorporate physical markers (trees, roads and railways) that outlive the average house. Within a few days of collection, maps showing relative mosquito densities can be produced by superimposing mosquito data onto the physical map. Gradients of mosquito numbers can be determined along sample transects or according to points on the map, and compared to human population densities to describe the heterogeneity in EIR. 


\section{The problems of non-temporal data}

Temporal variation in mosquito numbers and species composition is an inherent problem to RA because of its snapshot nature. Nevertheless, relative gradients in mosquito densities between areas do not change overmuch (Figure 1) and ratios between collection sites at any one time will reflect all other collections. When coupled to meteorological data and compared to published material, it should be possible to place the RA 'snapshot' into a reasonably predictable temporal context (Charlwood et al. 1995a).

\section{Malaria in the mosquito}

Parasites can be identified in the mosquito by a range of simple or sophisticated techniques. Asexual and early sexual stages are easily identified in the blood meals of recently fed females, and their prevalence is proportional to parasite prevalence in human blood samples (Figure 7, Gare and Billingsley unpublished). Oocysts on the dissected midgut of unfed and gravid females can be observed through a compound microscope or, with care, a good dissecting microscope. The proportion of parous mosquitoes with oocysts provides an indication of the proportion of feeds infective to mosquitoes (the infectious reservoir) (Haji et al. 1996; Charlwood et al. 1997; Graves et al. 1988; Muirhead-Thomson 1954). Sporozoite identification by microscopy is labour-intensive, and does not identify Plasmodium species, although this is not a problem where only one parasite species is present, and ELISA or PCR on stored mosquitoes requires time and cost investment. A recently introduced dipstick assay offers an important field tool for RA, providing the first method to identify salivary gland sporozoites to species that is not laboratory-based and reliant upon equipment (Ryan et al. 2002).

\section{Age structure of mosquito population}

Depending on the type of collection the abdominal condition of mosquitoes provides an indication of the age structure of the population and their degree of endophily (Figure 5). For example, if a similar number of gravid and recently fed mosquitoes are collected resting from inside houses this indicates that the species is endophilic and probably susceptible to control by residual insecticides. Conversely, if there are many blood-fed and few gravid mosquitoes in a collection then the species is largely exophilic. The relative proportions of blood-fed, semi-gravid and gravid insects also provide an indication of the duration of the oviposition cycle length (Gillies 1956). Not all collection methods sample the same fraction of the population. The indoor resting sample, for example, tends to under-sample recently emerged insects and cannot therefore be used to determine recruitment (Takken et al. 1998).

Recruitment can be assessed by dissection of the ovaries. Depending on the dispersal pattern of the species, one might expect a large number of young insects close to breeding sites and fewer but older insects at a distance. In an RA, simple dissections can provide estimates of comparative potential risk in space as well as time. In assessing risk at any particular time it is also important to know if the vector population(s) are increasing, declining or stable (Figure 3). This can be determined by dissection of unfed mosquitoes (Figure 2) for the presence of a mating plug, the ovarian stage and parity status. Plugs are found only in young females and suggest localized breeding (Gillies 1956; Charlwood, Vij and Billingsley 2000) and young females with early stage ovarioles also indicate breeding taking place. The presence of ovariolar sacs in parous females indicates that the mosquitoes returned to feed shortly 


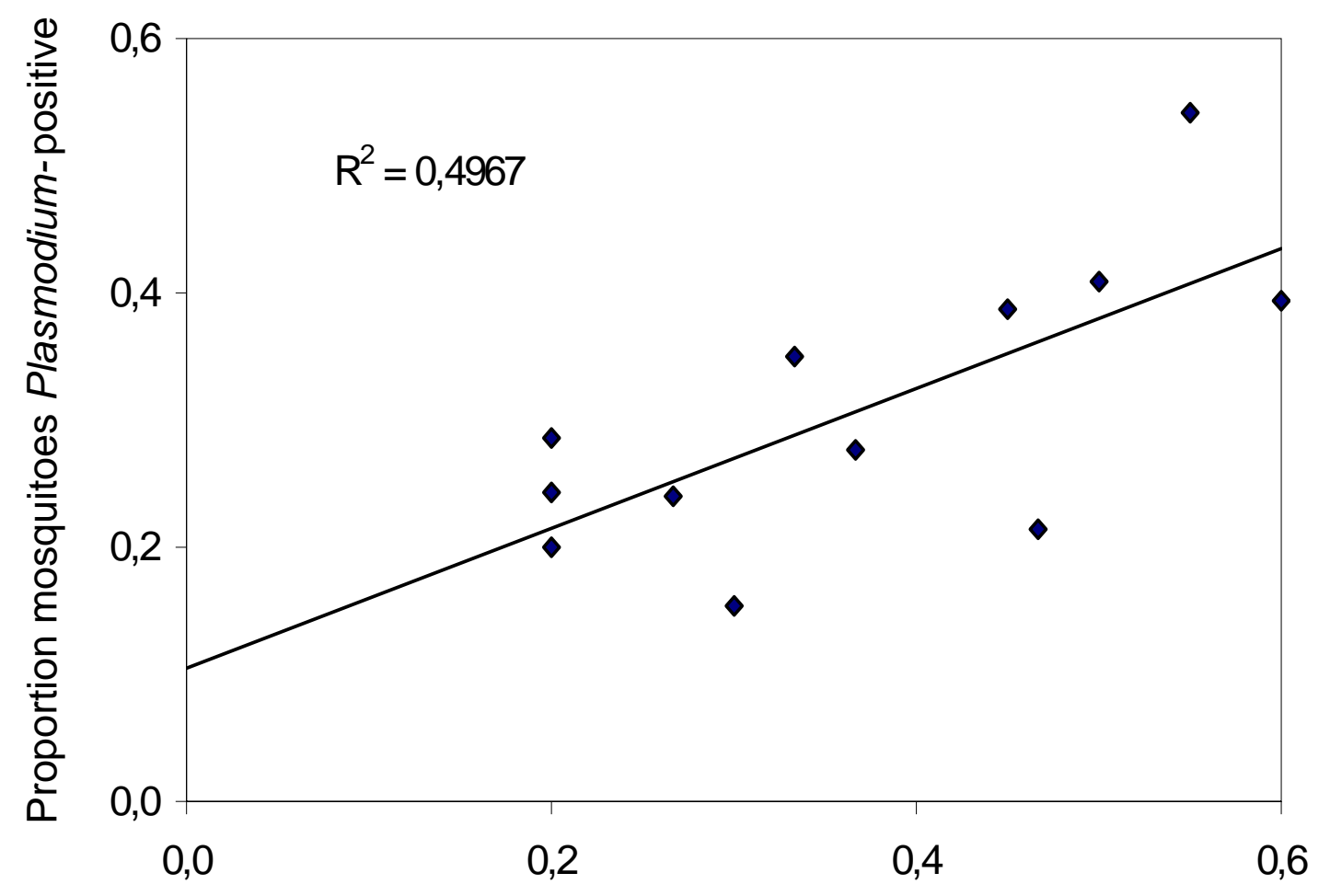

Proportion hosts Plasmodium-positive

Figure 7. Relationship between Plasmodium falciparum asexual-stage intensities in human blood smears and mosquito blood meals. For a limited period after feeding, mosquito blood meals contain all components of the host blood, including asexual and sexual stages of the malaria parasites. The proportion of blood meals containing parasites has a direct relationship with the proportion of infected individuals within a household, and can be used as an indicator of parasite prevalence. Each point on the graph represents samples taken from households of different numbers of individuals, the infected proportion of which varied. For each household, blood meals from at least 30 mosquitoes were sampled (Gare and Billingsley unpublished)

after oviposition and therefore oviposition sites are likely to be close to the feeding site. The relative proportions of parous females with and without sacs can be used to determine mean ovipostion cycle length.

Survival-rate estimation is an important epidemiological parameter that has received the attention of entomologists for many years. Estimates of survival rate from parity rates of samples collected over a short period of time are only applicable if the population is in a steady state (i.e. when recruitment and death rates are equal) (Charlwood et al. 1995a) and here, setting the RA into a temporal context is important. Alternatives to parous-rate estimations (e.g. time series analysis) all require longitudinal collections, placing them outside the RA remit, and have generally proven inapplicable to mosquito studies (Charlwood 1986; Charlwood, Vij and Billingsley 2000).

Whilst the proportion of a population parous may vary due to rapid changes in recruitment, the infection rate in parous insects changes more slowly. Combined parous and oocyst rates provide a suitable estimate of the proportion of feeds infectious to the mosquito (Charlwood, Vij and Billingsley 2000); by further including sporozoite rate, mosquito survival can also be estimated (Charlwood et al. 1995a; Beier and Koros 1991). Estimates of sporozoite rates are a needed for EIR 
determination and are therefore likely to be included in any RA protocol. The relative proportion of parous mosquitoes with oocysts and sporozoites provides an indication of survival rate, age structure and vector potential. A comparison of actual and potential sporozoite rate (determined by survival rate of the mosquito) indicates the potential for amplified transmission. Under steady-state conditions the relative proportion of each age group, as defined by parity status and sac stage (of parous females), is an indicator of mosquito survival rate. Large imbalances in any age category imply that the population is growing recruiting or ageing, or that representative samples of the different age groups are not being obtained.

\section{Mosquito behaviour}

The feeding behaviour, in particular the human-biting rate, is an important component of EIR estimation. Abdomens of blood-fed females dried onto filter paper can be used to identify host source by ELISA or dipstick ELISA (Savage et al. 1991; Charlwood et al. 2001), either of which can be performed under field conditions. By siting collections indoors or outdoors, the exophilic behaviour of mosquitoes can be determined, and coupled with knowledge of the biting cycle and local habits, used to refine risk assessment.

\section{Data management}

The availability and affordability of palmtop and laptop computers, and their great advantages in terms of accuracy and efficiency of data input (Forster and Snow 1992) places them as essential items in the RA repertoire. A core working practice can be envisaged that will serve several purposes. Spreadsheets or databases can be designed for manual and automated (e.g. GPS, data-logging devices, weather stations) data input, and simultaneous sample processing (through unique labelling). The spreadsheets on palmtops could then be processed into a complete database for full data analysis. Analysis would include EIR estimates, mapping, spatial and (limited) temporal variations in mosquito numbers, risk factors, mosquito species contributions to transmission, and best sites to target for control measures. Inputting data directly into computers allows immediate cross-checking of data and their rapid processing (Sutherst 1998). The outputs can be formulated to provide information for other projects (such as MARA), to be comparable with other studies, be tailored towards available software for such things as impact assessment (Hay, Snow and Rogers 1998), be used to validate statistical (Smith et al. 1995) or predictive (Lindsay, Parson and Thomas 1998) models, or improve the resolution of maps of vector abundance (Snow, Marsh and Le Sueur 1996) or transmission intensity (Graves et al. 1988). Finally, with standardized data gathering and input coupled to archived field material, an RA repository could provide stored samples and on-line databases for a range of collaborative studies.

It is also important, of course, to ensure that answers derived from an RA to a specific set of questions are used to improve control efforts. The utility of the data depends upon the scale of the RA, resources available and questions being addressed. At the larger scale RA may allow a focusing of resources, say to particular villages within a district. Even within 'low-density' villages the RA may identify foci of intense transmission appropriate for such targeted control strategies as house spraying or selective vaccination. 


\section{Rapid assessment: present and future}

The RA techniques described here are often applied by entomologists with or without RA interests; the techniques are all available, but have yet to be packaged into an assessment protocol. Given the increased scope for networking and data exchange, uniform methodology can be developed and results compared between areas. For example, if CDC miniature light traps are used to collect indoor biting vectors it behoves entomologists to use them in the same way (Mbogo et al. 1993; Mboera et al. 1998; Snow, Marsh and Le Sueur 1996). Broad use of the same techniques should provide hitherto unavailable ecological information on a continental scale; in Africa, in particular, the absence of such information makes it difficult to assess ecological trends within the An. gambiae complex. Meta-analysis from a number of sites could provide information on such things as species-specific dispersal rates under different ecological conditions. The techniques are available and the entomologists are there; what is required for the 'present' of RA is communication, coordination and application.

Once RA is established, its future looks brighter still. Numerous techniques that were developed over the past years, some in areas not related to malaria, can impact upon field studies in general and RA in particular. More use could be made of the mosquito blood meal to identify anti-CSP antibodies (exposure index in the humans), proteinase activities (time post feeding and therefore biting cycle), and human-feeding rate by dipstick assays. Plasmodium species-specific CSP can be detected in live mosquitoes (Billingsley et al. 1991) and the technique should be adaptable to determine low sporozoite rates in large mosquito samples as an alternative to ELISA or population screening (Shen et al. 1998). With the exception of polytene chromosome examination, there is no reliable field-based assay for identification of mosquitoes within a species complex. PCR-based approaches (Paskewitz and Collins 1990) require a laboratory, and DNA squashes (Hill, Urwin and Crampton 1991) have not lived up to their promise. It is possible that such differences in mosquito biology as attraction to specific odours or desiccation resistance can be exploited to identify separate species in simple ways. The equipment-driven side of RA also has an interesting future. Combined with data from weather stations (portable or regionally sited), GIS (Geographic Information Systems) information could be extrapolated to locate oviposition sites and enhance or validate predictive models from satellite data. A clockwork electrical supply, currently used to drive transistor radios, could allow microscopes, light traps and computers to be taken to remote sites without the need for generators (same for solar-powered $12 \mathrm{~V}$ battery packs). Identification of host odours can drastically improve the efficiency of mosquito traps as shown with $\mathrm{CO}_{2}$ and 1-octen-3-ol in mosquito traps in Florida (Takken and Kline 1989).

An explicit mosquito-based RA of malaria in the field has yet to be attempted. With current methodologies we urge field entomologists and malariologists to attempt some of the approaches outlined here. Their success will help develop an approach to malaria assessment that will be broadly applicable and can be implemented with limited training of local entomologists within a health-care setting.

\section{References}

Beier, J.C. and Koros, J.K., 1991. Visual assessment of sporozoite and bloodmeal ELISA samples in malaria field studies. Journal of Medical Entomology, 28 (6), 805-808. 
Billingsley, P.F., Hodivala, K.J., Winger, L.A., et al., 1991. Detection of mature malaria infections in live mosquitoes. Transactions of the Royal Society of Tropical Medicine and Hygiene, 85 (4), 450-453.

Billingsley, P.F., Medley, G.F., Charlwood, J.D., et al., 1994. Patterns of infection of Plasmodium falciparum in wild caught Anopheles mosquitoes. American Journal of Tropical Medicine and Hygiene, 51, 260-270.

Carabin, H., Escalona, M., Marshall, C., et al., 2003. Prediction of community prevalence of human onchocerciasis in the Amazonian onchocerciasis focus: Bayesian approach. Bulletin of the World Health Organization, 81 (7), 482490.

Charlwood, J.D., 1986. Survival rate variation of Anopheles farauti (Diptera: Culicidae) between neighbouring villages in coastal Papua New Guinea. Journal of Medical Entomology, 23 (4), 361-365.

Charlwood, J.D., Kihonda, J., Sama, S., et al., 1995a. The rise and fall of Anopheles arabiensis (Diptera: Culicidae) in a Tanzanian village. Bulletin of Entomological Research, 85, 37-44.

Charlwood, J.D., Qassim, M., Elnsur, E.I., et al., 2001. The impact of indoor residual spraying with malathion on malaria in refugee camps in eastern Sudan. Acta Tropica, 80 (1), 1-8.

Charlwood, J.D., Smith, T., Billingsley, P.F., et al., 1997. Survival and infection probabilities of anthropophagic anophelines from an area of high prevalence of Plasmodium falciparum in humans. Bulletin of Entomological Research, 87 (5), 445-453. [http://pest.cabweb.org/PDF/BER/BER87-5/445.pdf]

Charlwood, J.D., Smith, T., Kihonda, J., et al., 1995b. Density independent feeding success of malaria vectors in Tanzania. Bulletin of Entomological Research, 85, 29-35.

Charlwood, J.D., Vij, R. and Billingsley, P.F., 2000. Dry season refugia of malariatransmitting mosquitoes in a dry savannah zone of east Africa. American Journal of Tropical Medicine and Hygiene, 62 (6), 726-732.

Forster, D. and Snow, B., 1992. Using microcomputers for rapid data collection in developing countries. Health Policy and Planning, 7 (1), 67-71.

Garrett-Jones, C., 1964. Prognosis for interruption of malaria transmission through assessment of the mosquito's vectorial capacity. Nature, 204, 1173-1175.

Gillies, M.T., 1956. A new character for the recognition of nulliparous females of Anopheles gambiae. Bulletin of the World Health Organization, 15 (3/5), 451459.

Graves, P.M., Burkot, T.R., Carter, R., et al., 1988. Measurement of malarial infectivity of human populations to mosquitoes in the Madang area, Papua, New Guinea. Parasitology, 96 (Part 2), 251-263.

Gupta, S. and Snow, R.W., 1996. How do bednets influence the transmissibility of Plasmodium falciparum? Parasitology Today, 12 (3), 89-90.

Haji, H., Smith, T., Meuwissen, J.T., et al., 1996. Estimation of the infectious reservoir of Plasmodium falciparum in natural vector populations based on oocyst size. Transactions of the Royal Society of Tropical Medicine and Hygiene, 90 (5), 494-497.

Hay, S.I., Snow, R.W. and Rogers, D.J., 1998. Predicting malaria seasons in Kenya using multitemporal meteorological satellite sensor data. Transactions of the Royal Society of Tropical Medicine and Hygiene, 92 (1), 12-20. 
Hii, J.L., Smith, T., Mai, A., et al., 2000. Comparison between anopheline mosquitoes (Diptera: Culicidae) caught using different methods in a malaria endemic area of Papua New Guinea. Bulletin of Entomological Research, 90 (3), 211-219.

Hill, S.M., Urwin, R. and Crampton, J.M., 1991. A comparison of non-radioactive labeling and detection systems with synthetic oligonucleotide probes for the species identification of mosquitoes in the Anopheles gambiae complex. American Journal of Tropical Medicine and Hygiene, 44 (6), 609-622.

Killeen, G.F., McKenzie, F.E., Foy, B.D., et al., 2000a. The potential impact of integrated malaria transmission control on entomologic inoculation rate in highly endemic areas. American Journal of Tropical Medicine and Hygiene, 62 (5), 545-551.

Killeen, G.F., McKenzie, F.E., Foy, B.D., et al., 2000b. A simplified model for predicting malaria entomologic inoculation rates based on entomologic and parasitologic parameters relevant to control. American Journal of Tropical Medicine and Hygiene, 62 (5), 535-544.

Kilombero Malaria Project, 1992. The level of anti-sporozoite antibodies in a highly endemic malaria area and its relationship with exposure to mosquitoes. Transactions of the Royal Society of Tropical Medicine and Hygiene, 86 (5), 499-504.

Koella, J.C., 1991. On the use of mathematical models of malaria transmission. Acta Tropica, 49 (1), 1-25.

Le Sueur, D., Binka, F., Lengeler, C., et al., 1997. An atlas of malaria in Africa. Africa Health, 19 (2), 23-24.

Lindblade, K.A., Walker, E.D. and Wilson, M.L., 2000. Early warning of malaria epidemics in African highlands using Anopheles (Diptera: Culicidae) indoor resting density. Journal of Medical Entomology, 37 (5), 664-674.

Lindsay, S.W., Parson, L. and Thomas, C.J., 1998. Mapping the ranges and relative abundance of the two principal African malaria vectors, Anopheles gambiae sensu stricto and An. arabiensis, using climate data. Proceedings of the Royal Society of London. Series B. Biological Sciences, 265 (1399), 847-854.

Mathenge, E.M., Killeen, G.F., Oulo, D.O., et al., 2002. Development of an exposurefree bednet trap for sampling Afrotropical malaria vectors. Medical and Veterinary Entomolgy, 16 (1), 67-74.

Mathenge, E.M., Omweri, G.O., Irungu, L.W., et al., 2004. Comparative field evaluation of the Mbita trap, the Centers for Disease Control light trap, and the human landing catch for sampling of malaria vectors in western Kenya. American Journal of Tropical Medicine and Hygiene, 70 (1), 33-37.

Mboera, L.E., Kihonda, J., Braks, M.A., et al., 1998. Influence of centers for disease control light trap position, relative to a human-baited bed net, on catches of Anopheles gambiae and Culex quinquefasciatus in Tanzania. American Journal of Tropical and Medical Hygiene, 59 (4), 595-596.

Mbogo, C.N., Glass, G.E., Forster, D., et al., 1993. Evaluation of light traps for sampling anopheline mosquitoes in Kilifi, Kenya. Journal of the American Mosquito Control Association, 9 (3), 260-263.

Medley, G.F., Sinden, R.E., Fleck, S., et al., 1993. Heterogeneity in patterns of malarial oocyst infections in the mosquito vector. Parasitology, 106 (5), 441449. 
Minja, H., Schellenberg, J.A., Mukasa, O., et al., 2001. Introducing insecticide-treated nets in the Kilombero Valley, Tanzania: the relevance of local knowledge and practice for an information, education and communication (IEC) campaign. Tropical Medicine and International Health, 6 (8), 614-623.

Muirhead-Thomson, R.C., 1954. Factors determining the true reservoir of infection of Plasmodium falciparum and Wuchereria bancrofti in a West African village. Transactions of the Royal Society of Tropical Medicine and Hygiene, 48 (3), 208-225.

Paskewitz, S.M. and Collins, F.H., 1990. Use of the polymerase chain reaction to identify mosquito species of the Anopheles gambiae complex. Medical and Veterinary Entomolgy, 4 (4), 367-373.

Ribeiro, J.M., Seulu, F., Abose, T., et al., 1996. Temporal and spatial distribution of anopheline mosquitos in an Ethiopian village: implications for malaria control strategies. Bulletin of the World Health Organization, 74 (3), 299-305.

Ryan, J.R., Dave, K., Collins, K.M., et al., 2002. Extensive multiple test centre evaluation of the VecTest malaria antigen panel assay. Medical and Veterinary Entomolgy, 16 (3), 321-327.

Savage, H.M., Duncan, J.F., Roberts, D.R., et al., 1991. A dipstick ELISA for rapid detection of human blood meals in mosquitoes. Journal of the American Mosquito Control Association, 7 (1), 16-23.

Shen, J., Zhang, S., Xu, B., et al., 1998. Surveillance for low-level malaria. Transactions of the Royal Society of Tropical Medicine and Hygiene, 92 (1), 3-6.

Smith, T., Charlwood, J.D., Takken, W., et al., 1995. Mapping the densities of malaria vectors within a single village. Acta Tropica, 59 (1), 1-18.

Snow, R.W., Marsh, K. and Le Sueur, D., 1996. The need for maps of transmission intensity to guide malaria control in Africa. Parasitology Today, 12 (12), 455457.

Sutherst, R.W., 1998. Implications of global change and climate variability for vectorborne diseases: generic approaches to impact assessments. International Journal of Parasitology, 28 (6), 935-945.

Takken, W., Charlwood, J.D., Billingsley, P.F., et al., 1998. Dispersal and survival of Anopheles funestus and An. gambiae s.l. (Diptera: Culicidae) during the rainy season in southeast Tanzania. Bulletin of Entomological Research, 88 (5), 561566.

Takken, W. and Kline, D.L., 1989. Carbon dioxide and 1-octen-3-ol as mosquito attractants. Journal of the American Mosquito Control Association, 5 (3), 311316.

Teuscher, T., 1992. Household-based malaria control in a highly endemic area of Africa (Tanzania): determinants of transmission and disease and indicators for monitoring-Kilombero Malaria Project. Memorias Do Instituto Oswaldo Cruz, 87 (Suppl. 3), 121-130.

Thompson, R., Begtrup, K., Cuamba, N., et al., 1997. The Matola malaria project: a temporal and spatial study of malaria transmission and disease in a suburban area of Maputo, Mozambique. American Journal of Tropical Medicine and Hygiene, 57 (5), 550-9.

Thomson, M.C., Connor, S.J., Milligan, P., et al., 1997. Mapping malaria risk in Africa: what can satellite data contribute? Parasitology Today, 8, 313-318. 
Thomson, M.C., Connor, S.J., Milligan, P.J., et al., 1996. The ecology of malaria-as seen from Earth-observation satellites. Annals of Tropical Medicine and Parasitology, 90 (3), 243-264.

Trigg, J.K., Mbwana, H., Chambo, O., et al., 1997. Resistance to pyrimethamine/ sulfadoxine in Plasmodium falciparum in 12 villages in north east Tanzania and a test of chlorproguanil/dapsone. Acta Tropica, 63 (2/3), 185-189.

Wijesundera, M.D., Peiris, J.S., Ariyaratne, Y.G., et al., 1990. Antibodies to Plasmodium falciparum sporozoites following a malarial outbreak in a nonendemic area of Sri Lanka. Transactions of the Royal Society of Tropical Medicine and Hygiene, 84 (1), 35-39.

Woolhouse, M.E., Dye, C., Etard, J.F., et al., 1997. Heterogeneities in the transmission of infectious agents: implications for the design of control programs. Proceedings of the National Academy of Sciences of the United States of America, 94 (1), 338-342. 